\title{
Construção de uma linha guia para hipertensos e diabéticos na Atenção Primária à Saúde: experenciando uma abordagem multidisciplinar
}

\author{
Monique Dinizio dos Santos, Andrezza Lima Muricy
}

\section{RESUMO}

A Atenção Primária à Saúde configura-se como porta de entrada preferencial do SUS e tem atraído debates sobre a fragmentação da assistência às Doenças Crônicas Não Transmissíveis, o que resulta na dificuldade de garantia dos princípios de longitudinalidade e integralidade. Nesse contexto, o presente trabalho, objetiva relatar a experiência da construção de linha guia para o cuidado dos usuários com condições crônicas em uma unidade de saúde da família no município de CamaçariBA. Após diagnóstico situacional das condições crônicas no território estudado, a construção da linha guia teve como referencial teórico o modelo de cuidado das condições crônicas na Atenção Primária à Saúde, proposto por Mendes e adaptado à realidade local após discussões entre autoras. Consequentemente, a experiência proporcionou a reflexão da equipe quanto ao uso da estratificação de risco como ferramenta que possibilita o direcionamento mais adequado aos recursos materiais e humanos para o cuidado das condições crônicas.

Palavras-chave: Doenças não Transmissíveis; Hipertensão; Diabetes Mellitus; Fatores de Risco de Doenças Cardíacas.

\section{ABSTRACT}

Primary Health Care is configured as the preferred gateway to SUS and has attracted debates about the fragmentation of care for Chronic Non-Communicable Diseases, which results in the difficulty of guaranteeing the principles of longitudinality and integrality. In this context, this study aims to report the experience of building a guideline for users with chronic conditions in a family health unit in the city of Camaçari-BA. After the situational diagnosis of chronic care conditions in the studied territory, the construction of the guideline had as theoretical framework the model of care for chronic conditions in Primary Health Care, proposed by Mendes and adapted to the local reality after occurring among the authors. Consequently, an experience provided the team with a reflection on the use of risk stratification as a tool that enables the most appropriate targeting of material and human resources for the care of chronic conditions.

Keywords: Non-Communicable Diseases; Hypertension; Diabetes Mellitus; Heart Disease Risk Factors.

Revista da Rede APS 2021

Publicada em: 28/12/2021

DOI:10.14295/aps.v3i3.188

Monique Dinizio dos Santos (Fundação Estatal de Saúde da Família, Salvador, BA, Brasil)

Andrezza Lima Muricy (Fundação Estatal de Saúde da Família, Salvador, BA, Brasil)

Correspondência para:

Monique Dinizio Santos modinizio@hotmail.com

Andrezza Lima Muricy dezzamuricy@hotmail.com 


\section{INTRODUÇÃO}

Nas últimas décadas têm se observado transformações no processo de adoecimento e mortalidade da população brasileira (JÚNIOR e RAMALHO, 2015). Esse contexto tem desenhado novo cenário para atuação do Sistema Único de Saúde (SUS), exemplificado pela configuração de tripla carga de doença que envolve a persistência de doenças infecciosas, desafio das doenças crônicas e seus fatores de risco, e o crescimento das causas externas (MARINHO, 2016).

O perfil do adoecimento da população brasileira demonstra que as doenças cardiovasculares representam uma das causas mais relevantes de mortalidade e que no SUS são responsáveis por $30 \%$ dos internamentos. Em todo mundo, a Hipertensão Arterial Sistêmica (HAS) e Diabetes Mellitus (DM) apontam para maior risco cardiovascular (SOUZA; MENDONÇA, 2016).

A Atenção Primária à Saúde (APS) configura-se como porta de entrada preferencial do SUS e tem atraído debates sobre a fragmentação da assistência as Doenças Crônicas Não Transmissíveis (DCNT's), o que resulta na dificuldade de garantia de longitudinalidade e integralidade (OLIVEIRA, 2020).

Durante a atuação como Enfermeira Residente em uma Unidade Básica de Saúde (UBS) no município de Camaçari-BA, evidenciou-se um grande número de atendimentos a usuários com DCNT's, pessoas com hipertensão e/ ou diabetes, desvinculados das suas equipes de referência, via demanda espontânea, para renovação de receitas ou por agudização da condição.

Tentando descrever o perfil epidemiológico da unidade referida, identificou-se que as equipes desconheciam a magnitude, morbidade e prevalência da HAS e DM no território adscrito, além da inexistência de uma linha guia para estruturação da assistência desses usuários. Como consequência, notou-se uma não estruturação do atendimento a demanda espontânea e programada, conforme risco e necessidades do usuário, acarretando fragmentação da assistência e dificuldade do cuidado efetivo e contínuo, conforme princípios do SUS.

Diante do exposto, fica explícito a necessidade de reunir um conjunto específico e organizado de conhecimentos capazes de subsidiar a atenção às pessoas com hipertensão e/ou diabetes. Visando estruturar uma proposta destinada ao atendimento centrado na pessoa e em suas necessidades, utilizou-se como questão norteadora: Como organizar o cuidado integral e longitudinal às pessoas com DCNT's na APS?

Nesse contexto, o presente trabalho, objetiva relatar a experiência da construção de uma linha guia para o cuidado dos usuários com condições crônicas em uma USF no município de CamaçariBA.

\section{METODOLOGIA}

Trata-se de um relato de experiência sobre construção de linha guia para o cuidado longitudinal das condições crônicas em uma UBS situada na região metropolitana de Salvador com o objetivo de promover mudanças nas práticas assistenciais. Elaborado e pautado na multidisciplinaridade e no contexto de processo formativo do Programa de Residências Integradas da Fundação Estatal Saúde da Família (FESF) em parceria com a Fundação Osvaldo Cruz (FIOCRUZ).

Foi desenvolvido por profissionais da categoria de enfermagem, medicina e odontologia, inseridas em uma USF do município de Camaçari-BA, no período de abril de 2019 a fevereiro de 2020, e orientado por corpo pedagógico do programa. Os encontros para elaboração e aprofundamento do tema sucederam-se semanalmente e, em alguns momentos, foram necessários encontros extras, fora do horário administrativo.

A construção foi realizada em três etapas. A primeira consistiu na revisão de literatura. Com base nos estudos encontrados e após discussões geradas, sucedeu a segunda etapa: levantamento de hipertensos e diabéticos da população adscrita da USF; construção do instrumento para a estratificação do risco; elaboração do plano terapêutico e do 
fluxograma de atendimento. Posteriormente ocorreu o planejamento e realização do teste piloto, seguido de avaliação e apresentação dos resultados para profissionais da unidade.

\section{RESULTADOS E DISCUSSÃO}

Visando organizar a gestão do cuidado referente a atenção às pessoas com condições crônicas em UBS de Camaçari, foi levantado a necessidade de fomentar uma ferramenta clínica que sistematizasse o processo de trabalho. Apesar da grande quantidade de atendimentos voltado para essas condições, não foi identificado ações preventivas. Ainda, ficou explícito que havia dificuldade dos ACS em manter atualizados os dados referentes as famílias já cadastradas.

Desse modo, na primeira etapa para organização do cuidado, realizou-se um levantamento bibliográfico, a fim de obter informações relativas a dados objetivos e subjetivos essenciais no cuidado das condições crônicas, assim como buscar experiências de lugares que já possuíam o cuidado estruturado.

O referencial teórico utilizado para a sistematização da linha, foi o modelo de cuidado das condições crônicas na APS proposto por Mendes (2012), descrito no Caderno de Atenção Básica no 35 (BRASIL, 2014), correlacionado com a estratificação de risco proposta pela da Sociedade Brasileira de Cardiologia - SBC (SBC, 2019) e com o aprendizado em campo.

Durante o estudo e discussões das literaturas elencadas como guia, percebeu-se que para organizar o cuidado estruturado ao paciente com condição crônica na USF, e consequentemente o planejamento das ações, seria necessário realizar análise situacional do território, já que os indicadores epidemiológicos e o processo de territorialização são importantes ferramentas para a organização dos processos de trabalho e para prática clínica na APS (SANTOS et al., 2010).

Apesar da ausência de banco de dados prévio, constatou-se através do levantamento de prontuários que 491 pessoas eram hipertensas, 71 diabéticas e ainda, 157 eram hipertensas e diabéticas no território estimado de 6.000 pessoas. A taxa pode ser subestimada, pois simultaneamente a coleta de dados, alguns prontuários eram utilizados no atendimento clínico. Percebeu-se que informações importantes sobre a identificação dos usuários e de suas condições estavam ausentes ou desatualizadas.

Considerando a magnitude evidenciada pelos dados levantados e os impactos dessas condições crônicas para a saúde da população, a equipe percebeu a necessidade de se construir uma linha guia para garantir um atendimento efetivo e voltado para as necessidades desses indivíduos, a fim de garantir vinculação desses usuários com equipe de referência.

A estratificação da população é centro dos modelos de atenção à saúde, permitindo identificar as pessoas e grupos com necessidade de saúde parecidos, devendo esses serem atendidos por tecnologias e recursos específicos, obedecendo o agrupamento segundo o risco (MENDES, 2012). Corroborando com isso, as autoras decidiram usar a estratificação de risco como ferramenta de decisão clínica para planejamento do cuidado. Elaborou-se dois instrumentos de estratificação de riscos: usuários hipertensos com ou sem diabetes, e para somente diabéticos; e posteriormente $\mathrm{o}$ instrumento do plano terapêutico.

Os instrumentos possibilitaram a avaliação subjetiva e objetiva da condição crônica, e após o preenchimento, foi possível a estratificação de risco e definição do plano de cuidado. A estratificação constitui-se como importante ferramenta para a organização da assistência, pois possibilita a equipe dimensionar os cuidados e atenção que se devem dar a pessoas, à medida que se reconhece que elas possuem diferentes graus de riscos / vulnerabilidades (BRASIL, 2014).

De acordo com Mendes (2012) as metodologias de estratificação de riscos de uma população podem envolver classificações que coordenem simultaneamente dois tipos de variáveis: severidade da condição crônica estabelecida e capacidade de autocuidado que considera aspectos socioeconômicos-culturais, o grau de 
confiança e o apoio que as pessoas têm para cuidar de si mesmas.

Apesar de já existir métodos que dispõe os riscos em estratos, ainda não há instrumentos voltados para estratificação de risco cardiovascular adaptado e com boas evidências científicas para a realidade brasileira (BRASIL, 2016). Logo, durante as discussões, considerando o cuidado multiprofissional e tendo como base a estratificação de risco segundo Mendes (2012), definiu-se que o instrumento possuiria quatro núcleos de abordagem: 1- identificação do paciente e condição clínica, 2- severidade da doença crônica, 3- capacidade do autocuidado e 4avaliação da saúde bucal.

A primeira parte do instrumento contempla os dados de identificação; condição clínica e alguns dados clínicos do pacientem como idade, altura, peso, pressão arterial (PA) e hemoglicoteste (HGT).

A severidade da doença crônica avalia variáveis relacionadas ao risco cardiovascular do indivíduo em 10 anos. Considerando os critérios de risco da Sociedade Brasileira de Cardiologia SBC (SBC, 2019), foram contemplados os seguintes dados: sexo/idade; histórico familiar de doença cardiovascular; hábitos de vida; marcadores laboratoriais; Índice de Massa Corpórea (IMC), circunferência da medida abdominal. Somado a essas variáveis, ainda é necessário identificar se há lesão em órgãosalvo e complicações microvasculares/ macrovasculares (PRÉCOMA et al., 2019). Após correlacionar esses dados com níveis pressóricos e/ou glicêmicos, determina-se os estratos que definem os riscos em: sem risco, baixo, moderado, alto e muito alto (BRASIL, 2014; PRÉCOMA et al., 2019).

A avaliação da capacidade do autocuidado é essencial porque as pessoas têm diferentes necessidades. Essa avaliação tem caráter dinâmico e é em boa parte subjetiva, pois depende da percepção da equipe de saúde acerca do contexto e da postura da pessoa diante de sua condição, assim como da interferência dos determinantes sociais em saúde (DSS) (BRASIL, 2014).
Após discussão, considerando que não há instrumento validado para avaliar a capacidade de autocuidado, e ainda em consonância com a realidade, foram definidas perguntas direcionadas acerca de três grandes grupos: compreensão sobre condição e adesão terapêutica; ausência de apoio familiar e/ou dificuldades socioeconômicas em relação sua condição e comorbidades associadas que dificultem o autocuidado, onde qualquer situação positiva será classificada como insuficiente.

As equipes de saúde devem entender o papel das doenças crônicas na etiologia das doenças bucais. Sendo assim, deve ocorrer com frequência a avaliação da saúde bucal, com o intuito de rastrear e tratar as condições, assim como a instituição de cuidados preventivos. A avaliação da saúde bucal, levou em consideração a identificação de sinais de alerta e manifestações bucais associadas às comorbidade estudas, trazidas por Cavalcanti (2010): sangramento, dor, abcesso, uso de prótese dentária, xerostomia, candidoses, doença periodontal e sialorreia.

Avaliar em conjunto o grau de severidade da doença crônica, a capacidade de autocuidado e a saúde bucal, objetivou conhecer melhor as necessidades de cuidado de cada indivíduo, e assim definir diretrizes clínicas, o tipo de atenção e permitiu planejar uma ação de saúde específica para cada grupo populacional do estrato.

No instrumento construído, a estratificação do paciente resultou em 5 estratos diferentes, que subsidiou a gestão da clínica por meio do plano terapêutico (Quadro 1). Dessa forma, as pessoas com condição crônica de menores riscos tinham sua condição centrada em tecnologias de atenção compartilhadas em atividades de grupo, enquanto aquelas com condições de alto e muito alto risco, tinham uma presença mais significativa de atenção multiprofissional com ação interdisciplinar na construção de Plano Terapêutico Singular (PTS).

O PTS proposto, concordou com o modelo proposto por Mendes (2012), ao estabelecer consultas, visitas e participação em grupos de 
educação e promoção de saúde anualmente, semestralmente ou trimestralmente de acordo com necessidade e riscos, pela equipe multidisciplinar - enfermeiro, dentista, médico e profissionais do Núcleo Ampliado de Saúde da Família e Atenção Básica (NASF-AB).

Quadro 1 - Plano terapêutico que orienta o tipo de atenção profissional necessária. Ilustra que para cada estrato de risco há uma recomendação de saúde específica predominante.

\begin{tabular}{|c|c|c|}
\hline NÍVEL DE AÇÃO & AÇÃO DE SAÚDE & SEGUIMENTO \\
\hline ESTRATO 5 & Gestão de Caso & $\begin{array}{l}\text {-Grupo Você no controle, } 3 \text { vezes ao ano } \\
-03 \text { consultas médicas anualmente* } \\
-02 \text { consultas de enfermagem anualmente* } \\
-02 \text { consultas odontológicas anualmente } \\
\text {-PTS } \\
\text { * Considerar atendimento domiciliar. }\end{array}$ \\
\hline ESTRATO 4 & Atenção individual & $\begin{array}{l}\text {-Grupo Você no controle, } 2 \text { vezes ao ano } \\
\text {-01 consulta médica anualmente } \\
-01 \text { consulta de enfermagem anualmente } \\
-02 \text { consultas odontológicas anualmente }\end{array}$ \\
\hline ESTRATO 3 & $\begin{array}{c}\text { Atenção individual / } \\
\text { compartilhada em atividade } \\
\text { de grupo }\end{array}$ & $\begin{array}{l}\text { - Grupo Você no controle, } 1 \text { vez ao ano } \\
-01 \text { consulta médica anualmente } \\
-01 \text { consulta de enfermagem anualmente } \\
-02 \text { consultas odontológicas anualmente }\end{array}$ \\
\hline ESTRATO 2 & $\begin{array}{l}\text { Atenção compartilhada em } \\
\text { atividade de grupo }\end{array}$ & $\begin{array}{l}-01 \text { consulta anual (medicina ou enfermagem) } \\
-01 \text { consulta odontológica semestral } \\
\text {-Grupo Você no controle a cada } 6 \text { meses }\end{array}$ \\
\hline ESTRATO 1 & $\begin{array}{l}\text { Grupos de educação e } \\
\text { promoção a saúde }\end{array}$ & $\begin{array}{l}\text { OFERTA DE: } \\
\text {-Grupo Batendo pernas } \\
\text {-Grupo de Auriculoterapia } \\
\text {-Grupo Bem-me-quero } \\
\text {-Horta Comunitária } \\
\text {-Grupo você no controle }\end{array}$ \\
\hline
\end{tabular}

Fonte: Elaborado pelas autoras.

Ainda, dentro do seguimento de cada estrato do plano terapêutico, a depender da descompensação da condição, existia conduta individualizada e, se necessário, maior oferta de uma atenção individualizada/consulta.

O passo subsequente para sistematizar o cuidado foi a construção da linha guia, que se estruturou por meio de um fluxograma no qual definiu o acesso e etapas da assistência dos usuários com condições crônicas (Figura 1).

O planejamento do teste piloto aconteceu durante o mês de novembro de 2019. Assim, guiado por discussões, decidiu-se a criação do grupo "Você no controle", que seria destinado a realizar estratificação de risco dos usuários, além de proporcionar um espaço de cuidado apoiado, através de roda de conversa. Estabeleceu que o teste piloto seria realizado com 18 usuários que residiam em três ruas da área de abrangência da unidade.

$\mathrm{Na}$ aplicação do teste piloto, compareceram 08 usuários. Participaram desse momento, as autoras, nutricionista, e preceptores do núcleo de medicina e do NASF-AB. Na ocasião, seguimos programação planejada: identificação do usuário, abordagem das condições crônicas e do espaço promovido, conversa sobre alimentação saudável e posteriormente os profissionais presentes realizaram a aplicação do instrumento individualmente com o usuário. 
Os usuários elogiaram o espaço de cuidado que estava sendo promovido, e desejavam que ele se perpetuasse, melhorando a assistência e o acesso. As autoras consideraram o espaço potente para a construção da educação em saúde, além de proporcionar um apoio ao autocuidado, a partir da troca de experiências. Após a aplicação, o instrumento foi reavaliado e

\section{CONSIDERAÇÕES FINAIS:}

O trabalho desenvolvido buscou garantir os princípios de longitudinalidade, integralidade e universalidade do SUS, com o objetivo de evitar complicações clínicas e socioeconômicas a respeito das condições crônicas. Os achados demonstram a importância da elaboração de uma linha guia, visto que a construção possibilitou a avaliação do risco do usuário e as estratégias que devem ser adotadas, contribuindo, portanto, para menor taxa de mortalidade.

Durante a criação da linha, algumas limitações foram encontradas, tais como: equipamentos de trabalho e rede de internet para aprofundamento teórico acerca do tema, dificuldade na coleta de dados em prontuários e a mudança da rotina de trabalho para o enfrentamento da COVID-19, que impactou diretamente na implantação do cuidado programado.

Como potencialidade, ressalto que a proposta pode subsidiar a atuação da equipe mínima num contexto interdisciplinar, colaborando diretamente na organização dos processos de trabalho entre equipes, no reconhecimento e contemplação da pessoa dentro da complexidade de todos os aspectos que a envolve. Além disso, contribuirá para a fundamentação das decisões em equipe quanto à previsão de atendimentos individuais na agenda, bem como o planejamento de atividades coletivas e atendimentos domiciliares.

Enquanto profissional de saúde, a experiência proporcionou-me a reflexão e mudanças acerca do cuidado efetivo ao paciente com condição crônica. Ainda, permitiu o aprofundamento de tecnologias que o enfermeiro pode lançar mão durante as consultas, como ferramenta readaptado. As alterações foram quanto a reformulação das perguntas da avaliação do autocuidado.

Em 04 de fevereiro de 2020, a linha guia foi apresentada para todos os profissionais da unidade. Ainda, foi disponibilizado para o serviço, na forma digital.

indispensável na assistência, melhoria da qualidade de vida desses usuários e na gestão dos processos de trabalho, a exemplo da estratificação de risco, visto que possibilita o direcionamento mais adequado dos recursos materiais e humanos.

Para o SUS a proposta contribuiu no entendimento de que a estratificação de risco da população possibilita a organização da rede de atenção à saúde, assim como na prestação de assistência efetiva, com custos, lugar e qualidade correta. Ainda, alerta para necessidade de educação permanente das equipes acerca dos processos de trabalho e da necessidade de cadastramento de toda população, para que assim haja responsabilização da ESF pela saúde desses indivíduos.

A experiência atenta para a maior necessidade de envolvimento dos governantes das diversas esferas e dos profissionais de saúde na assistência ao paciente com condição crônica. Compete aos gestores: incentivar e integrar as políticas intersetoriais que contemplam os DSS; desenvolver e ampliar ações voltadas para educação continuada dos recursos humanos do SUS e manter a integração dos serviços de saúde com as instituições de ensino superior, incentivando pesquisas, desenvolvimento científico e tecnológico no cuidado desses usuários. Espera-se que os gestores sigam firmes na formulação de estratégias e no controle da execução das políticas públicas de saúde, fortalecendo assim, a Rede de Atenção à Saúde das Pessoas com Doenças Crônicas. 


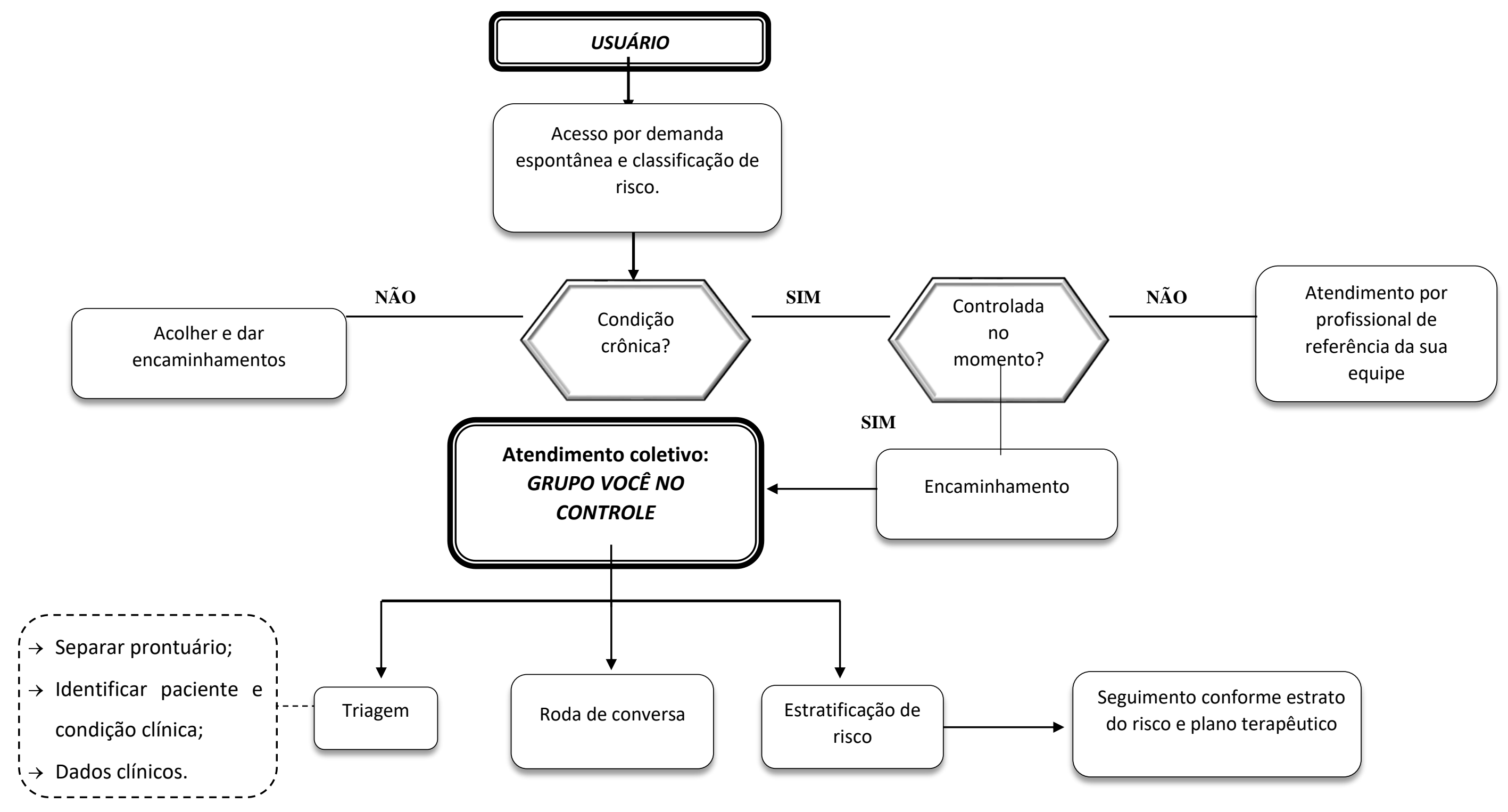




\section{REFERÊNCIAS}

BRASIL. Ministério da Saúde. Protocolos da atenção básica: condições crônicas não-transmissíveis: risco cardiovascular / Ministério da Saúde, Instituto Sírio Libanês de Ensino e Pesquisa. - Brasília: Ministério da Saúde, 2016.

BRASIL. Ministério da Saúde. Secretaria de Atenção à Saúde. Departamento de Atenção Básica. Estratégias para o cuidado da pessoa com doença crônica / Ministério da Saúde, Secretaria de Atenção à Saúde, Departamento de Atenção Básica. - Brasília: Ministério da Saúde, 2014.

CAVALCANTI, A. M. Diabete Melito Tipo 2: diretriz de atenção à pessoa com Diabete Melito Tipo 2. Curitiba, PR: Secretaria Municipal da Saúde, 2010.

JÚNIOR, J. B.; RAMALHO, W. M. Cenário epidemiológico do Brasil em 2033: uma prospeç̧ão sobre as próximas duas décadas /. - Rio de Janeiro: Fundação Oswaldo Cruz, 2015. 16 p.- (Textos para Discussão; n. 17)

MARINHO, F.; PASSOS, V. M. A.; FRANCA, E. B. Novo século, novos desafios: mudança no perfil da carga de doença no Brasil de 1990 a 2010. Epidemiol. Serv. Saúde, Brasília, v. 25, n. 4, p. 713-724, Dec. 2016.

MENDES, E. V. O cuidado das condições crônicas na atenção primária à saúde: o imperativo da consolidação da estratégia da saúde da família. Brasília, OPAS, 2012.

OLIVEIRA, J. H.; SOUZA, M. R.; NETO, O. L. M. Enfrentamento das doenças crônicas não transmissíveis na atenção primária à saúde em Goiás: estudo descritivo, 2012 e 2014. Epidemiol. Serv. Saúde, Brasília, v. 29, n. 5, e2020121, 2020.

PRÉCOMA, D. B. et al. Atualização da diretriz de prevenção cardiovascular da Sociedade Brasileira de Cardiologia-2019. Arquivos Brasileiros de Cardiologia, v. 113, n. 4, p. 787-891, 2019.

SANTOS, A. L; RIGOTTO, R. M. Território e territorialização: incorporando as relações produção, trabalho, ambiente e saúde na atenção básica à saúde. Trab. educ. saúde (Online), Rio de Janeiro, v. 8, n. 3, p. 387 406, nov. 2010.

SOUZA, K. M. M; MENDONÇA, F. A. C. Estratificação de risco de hipertensos e diabéticos em uma unidade de atenção primária de Fortaleza-Ceará. Revista tendências da enfermagem profissional, 2016. 